\title{
Systematic Differences in Effect Estimates Between Observational Studies and Randomized Control Trials in Meta-Analyses Combining Both Study Designs in Nephrology
}

Miho Kimachi ( $\nabla$ cuisse.de.nymph@gmail.com )

Kyoto University

Akira Onishi

Kobe University

Aran Tajika

Kyoto University

Kimihiko Kimachi

Kyoto University

Toshi Furukawa

Kyoto University

\section{Research Article}

Keywords: RCTs, ROR, nephrology

Posted Date: November 30th, 2020

DOI: https://doi.org/10.21203/rs.3.rs-109178/v1

License: (c) (i) This work is licensed under a Creative Commons Attribution 4.0 International License.

Read Full License

Version of Record: A version of this preprint was published at Scientific Reports on March 17th, 2021. See the published version at https://doi.org/10.1038/s41598-021-85519-5. 


\section{Abstract}

The limited availability of randomized controlled trials (RCTs) in nephrology undermines causal inferences in meta-analyses. Systematic reviews of observational studies have grown more common under such circumstances. We conducted systematic reviews of all comparative observational studies in nephrology from 2006 to 2016 to assess the trends in the past decade. We then focused on the metaanalyses combining observational studies and RCTs to evaluate the systematic differences in effect estimates between study designs using two statistical methods: by estimating the ratio of odds ratios (ROR) of the pooled OR obtained from observational studies versus those from RCTs and by examining the discrepancies in their statistical significance. The number of systematic reviews of observational studies in nephrology had grown by 11.7 -fold in the past decade. Among 56 records combining observational studies and RCTs, ROR suggested that the estimates between study designs agreed well (ROR: $1.05,95 \%$ confidence interval: 0.90-1.23). However, almost half of the reviews led to discrepant interpretations in terms of statistical significance. In conclusion, the findings based on ROR might encourage researchers to justify the inclusion of observational studies in meta-analyses. However, caution is needed as the interpretations based on statistical significance were less concordant than those based on ROR.

\section{Introduction}

Randomized controlled trials (RCTs) provide high levels of evidence because they can minimize threats to internal validity. However, it is difficult to conduct RCTs in certain situations, such as with participants with serious complications, interventions with ethical constraints (e.g. surgical procedures) and serious adverse effects ${ }^{1-4}$. In particular, RCTs in nephrology have been limited because patients with kidney diseases generally have a number of complications ${ }^{5-9}$. When the number of available RCTs is insufficient, meta-analyses restricted to RCTs can be misleading ${ }^{10,11}$. Authors might then be justified in including observational studies ${ }^{3,12}$. Observational studies can reflect real world practices and have superior generalizability in comparison with RCTs under ideal conditions. However, the GRADE system reports that nonrandomized studies constitute only a low level of evidence due to many threats to internal validity ${ }^{13}$. Discrepancies in the findings between observational studies and RCTs can be caused by differences in sample size, confounding factors, several biases such as selection bias, publication bias, and follow-up period $^{14,15}$. In particular, unmeasured confounding factors can hamper causal inferences between the exposure and outcome ${ }^{16,17}$. Despite such controversy, several previous studies have reported that there were no differences in the risk estimates obtained from meta-analyses of observational studies in comparison with from those from RCTs ${ }^{14,18,19}$. However, the evidence has not been sufficiently established in nephrology. Further, recent meta-analyses comparing observational studies with RCTs based their conclusions on the ratio of odds ratios (ROR) between the pooled OR derived from observational studies and those derived from RCTs, while most clinical studies generally interpret efficacy based on the statistical significance ${ }^{18-22}$. 
Therefore, in the present study, we aimed to 1) assess the trends and characteristics of systematic reviews of observational studies in nephrology in the past decade; and 2) quantify systematic differences in effect estimates between observational studies and RCTs in meta-analyses using two statistical methods: ROR and discrepancies in statistical significance between the two study designs among metaanalyses combining observational studies and RCTs.

\section{Methods}

\section{Literature search and selection of studies}

The literature searches were conducted in January 2017 using EMBASE and MEDLINE. We searched studies published from January 2006 to December 2016 with no language limitation. The search strategy was developed with the assistance of a medical information specialist. The search strategy included key words related to 'observational study', 'systematic review', and 'kidney disease' (see Supplement Table 1). Search terms relevant to this review were collected through expert opinion, literature review, controlled vocabulary-including Medical Subject Headings (MeSH) and Excerpta Medica Tree-and a review of the primary search results. The titles and abstracts were screened independently by two authors (M.K, K.K) and were excluded during screening if they were irrelevant to our research question or duplicated. Studies suspected of including relevant information were retained for full text assessment using inclusion and exclusion criteria. If more than one publication of one study existed, we grouped them together and adopted the publication with the most complete data. The present study was conducted according to a protocol prospectively registered at PROSPERO (CRD42016052244).

\section{Evaluation of the characteristics of the systematic reviews of observational studies}

We included systematic reviews of all comparative observational studies in nephrology to assess the trends and characteristics of systematic reviews of observational studies in nephrology in the past decade. We included systematic reviews published from 2006 to 2016 to assess the influence of reporting assessment tools including PRISMA (Preferred Reporting Items for Systematic Reviews and Meta-analyses) published in 2009 and the risk of bias (RoB) tools including the Newcastle-Ottawa Scale (NOS) in 2007 and the Cochrane Risk of Bias Assessment Tool for Non-Randomized Studies of Interventions (ACROBAT-NRSI) in 2014. We selected studies of kidney diseases based on the following two criteria:

1. We included studies on participants with kidney diseases. Kidney diseases were defined as diseases that occurred in the renal parenchyma, such as acute or chronic kidney injury, kidney neoplasms, and nephrolithiasis, based on the MeSH search builder of the term 'Kidney Diseases'. Studies were excluded if they had participants with extra-renal diseases including ureteral diseases, urethral diseases, and urinary bladder diseases.

2. We included studies with primary outcomes related to kidney diseases. We used the same definition of kidney diseases as above. We excluded studies wherein kidney diseases were treated as a 
composite outcome (e.g. composite outcome of kidney, pancreas, and liver cancers).

We described the characteristics of systematic reviews of observational studies as follows:

1. The number of published systematic reviews of observational studies per year

2. Country of first author's institution

3. Designs of observational studies

- Cohort studies included prospective and retrospective cohort studies

- Case-control studies included ordinary and nested case-control studies.

4. Number of primary studies included in each review

5. Cause of kidney injury

6. Funding source

- Included support from both public institutions and industrial firms

7. Whether each review assessed the RoB

8. Whether each review performed a reporting assessment

- Reporting assessment tools were PRISMA, MOOSE (Meta-analyses of Observational Studies in Epidemiology), and QUAROM (The Quality of Reporting of Meta-analyses). STROBE (Strengthening the Reporting of Observational Studies in Epidemiology), CONSORT (Consolidated Standards of Reporting), and others were excluded.

\section{Comparison of effect estimates between observational studies and RCTs in meta-analyses combining both types of studies}

To compare the effect estimates between study designs, we focused on the meta-analyses combining observational studies and RCTs that compared two specific interventions. We included non-randomized studies, such as cohort, case-control, cross-sectional, and controlled trials that use inappropriate strategies of allocating interventions (sometimes called quasi-randomized studies), as observational studies $^{23}$. We expressed the quantitative differences in effect estimates for primary efficacy outcomes between study designs, taking the ROR ${ }^{24}$. Further, we assessed discrepancies in the statistical significance between study designs. The absence of discrepancies, which represents agreement between efficacy and effectiveness, was defined as follows: 1) both study types were significant with the same direction of point estimates, and 2) both study types were not significant. In contrast, the presence of discrepancies was defined as follows: 1) one study type was significant while the other type was not significant, and 2) both study types were significant, although the point estimates had the opposite direction ${ }^{24}$. We performed the assessment of the methodological quality of these meta-analyses combining both types of studies using AMSTAR (assessment of multiple systematic reviews) appraisal 
tool $^{25}$. Two review authors (M.K, A.O) independently graded each review for rating overall confidence as high, moderate, low, and critically low.

\section{Data extraction}

Two authors (M.K, K.K) collected the above-mentioned characteristics of systematic reviews of observational studies. Further, we also determined the effect size for the primary outcome of the primary articles. All relevant data related to the comparison of effect estimates between study designs were independently reconfirmed by two authors (A.O, A.T) using standard data extraction forms.

\section{Statistical analyses}

We described the baseline characteristics of systematic reviews of observational studies using means (standard deviation [SD]) for continuous data with a normal distribution, medians (interquartile range [IQR]) for continuous variables with skewed data, and proportions for categorical data.

For the comparison of effect estimates between observational studies and RCTs in meta-analyses combining both types of studies, we extracted relative risks or risk ratios (RR), OR, hazard ratios (HR), standardized mean differences (SMD), and mean differences (MD) of each primary study and their standard errors (SE) or $95 \%$ confidence interval $(\mathrm{Cl})$ from reviews as outcome measures. If an OR was not reported in a review, we recalculated the OR by extracting the number of events and non-events in both the intervention and control groups from a review or the primary study itself. If the number of events or non-events was 0 , we added 0.5 to all cells of each result ${ }^{23}$. If we could not determine the number of events or non-events from a review or primary articles to calculate the OR, we substituted original outcome measures, such as RR and HR, instead of OR. Additionally, SMD and MD were converted to OR based on a previous study ${ }^{26}$. The $\mathrm{SE}$ and $95 \% \mathrm{Cl}$ were calculated in accordance with previous studies $^{22,24}$. Further, if the reviews did not report effect sizes separately for two designs, we synthesized the results obtained from primary articles. If positive outcomes such as survival were adopted, the OR comparing the intervention with control were coined. Additionally, if ordinary or older interventions were included in the numerator of the OR, those OR were also coined. If several outcomes were reported, we used the first outcome that was described in the paper.

We estimated the difference in the primary efficacy outcomes between study designs by calculating the pooled ROR with the $95 \% \mathrm{Cl}$ using a two-step approach ${ }^{27}$. First, the ROR was estimated with the OR obtained from observational studies and RCTs in each review using random effects meta-regression. Second, we estimated the pooled ROR with the $95 \% \mathrm{Cl}$ across reviews with a random-effects model. Further, we performed sensitivity analysis using fixed effect model. If the ROR was more than 1.0, this would indicate that the OR from observational studies were larger than those from RCTs. Heterogeneity was estimated using $1^{2}$ test $^{23} . I^{2}$ values of $25 \%, 50 \%$ and $75 \%$ represent low, medium and high levels of heterogeneity. 
Further, we examined the association between discrepancies in statistical significance of each design in accordance with above-mentioned definitions and risk factors using a multiple logistic regression model, adjusted for difference in the number of primary articles between study designs, publication year, countries of first authors, pharmacological intervention, adjustment for confounding factors, and methodological quality of systematic reviews based on rating overall confidence of AMSTAR tool.

All statistical analyses were performed using STATA 16.0 (StataCorp LLC, College Station, TX, USA).

\section{Results}

\section{Study flow diagram}

The PRISMA flow diagram (see Fig. 1) shows the study selection process. Of 5,547 records identified through database searching, we screened the titles and abstracts of the 3,994 records remaining after removing duplicates and ultimately obtained 613 records. After a full-text review, we included a total of 477 records for the description of characteristics of systematic reviews of observational studies. Further, of the 114 records that combined both observational studies and RCTs, 56 were eligible for the evaluation of quantitative systematic differences in effect estimates of meta-analyses between observational studies and RCTs (see Supplement Table 2).

\section{Trends over the past decade and description of study characteristics}

We summarized the baseline characteristics of 477 nephrology systematic reviews of all comparative observational studies (see Table 1). The number of systematic reviews of observational studies in nephrology has increased by 11.7-fold in 2016 compared to 2006. In particular, the number of publications from China as well as the United States of America and European countries has increased (see Supplement Table 3). As shown in Table 1, most of the reviews dealt with topics related to therapies for patients with acute kidney injury, malignancy, end-stage renal diseases, and renal transplantation, aside from basic research. As for the eligible designs of observational studies, $67.1 \%$ records included cohort studies, and $33.8 \%$ included case-control studies. Of the 82 reviews related to basic research, 75 $(91.5 \%)$ included case-control studies. Case series and before-after studies without comparisons were excluded in many studies. NOS was the most frequently used tool for assessing the risk of bias. ACROBAT-NRSI was used in only $0.8 \%$ of records.

\section{Comparison of qualitative systematic differences in effect estimates between observational studies and RCTs in meta-analyses combining both types of studies}

Fifty-six meta-analyses combining both observational studies and RCTs were eligible for the analyses. A total of 418 observational studies and 204 RCTs were included, and the median number per metaanalysis was 7 ( 2.5 to 10 ) observational studies and 3 (2 to 5) RCTs. Almost reviews indicated a critically low quality (see Supplement Table 4). 
We compared the effect estimates of primary outcomes between study designs using the ROR with the $95 \% \mathrm{Cl}$. No significant differences were noted in the effect estimates between study designs (ROR 1.05, $95 \% \mathrm{Cl} 0.90$ to 1.23 ) (see Fig. 2). There was moderate heterogeneity $\left(\mathrm{I}^{2}=47.5 \%\right)$. Additionally, the result obtained using the fixed effect model was almost similar with that obtained using the random effect model (ROR $0.98,95 \% \mathrm{Cl} 0.89$ to 1.07). Of the 56 studies, 2 reviews showed that observational studies had significantly larger effects than RCTs (ROR $>1.0)$, while 6 showed that observational studies had significantly smaller effects than RCTs $(R O R<1.0)$. The remaining 48 reviews indicated no significant differences between the study designs.

Of the 56 studies, 29 reviews showed no discrepancy in terms of statistical significance (14 reviews; significant in the same direction as the point estimates, 15 reviews; neither significant), while 27 reviews showed some discrepancy (all 27 studies: one significant and the other not significant). No review showed statistical significance in the opposite direction of the point estimates. Table 2 shows a comparison of the baseline characteristics between the presence and absence of discrepancies. In addition, we explored the factors associated with discrepancies (see Table 3), but no significant association was noted for any covariate, in particular, difference in the number of papers between observational studies and RCTs (OR 1.10, 95\% $\mathrm{Cl} 0.99$ to 1.23 ).

Further, comparing the results from ROR and the distribution of discrepancies of statistical significance, of 48 records (85.7\%) that indicated the non-significance of the ROR, 20 (35.7\%) showed discrepancies in statistical significance (see Table 4).

\section{Discussion}

The findings of the present study indicated that the numbers of systematic reviews of observational studies in nephrology have dramatically increased in the past decade, especially from China and the United States of America. Around $60 \%$ of the reviews assessed the risk of bias, mostly using the NOS. A comparison of effect estimates between observational studies and RCTs in meta-analyses combining both types of studies revealed that the effect estimates from observational studies were largely consistent with those from RCTs. However, when interpreted in terms of statistical significance, almost half of the reviews led to discrepant interpretations.

Observational studies generally have larger sample sizes and better represent real-world populations than RCTs. Nevertheless, confounding factors, especially confounding by indication, often disturb the precise assessment of causal inference and establishment of high levels of evidence ${ }^{28-31}$. The quality of the evidence based on observational studies might depend on how confounding factors are controlled. Adjustment using appropriate techniques, including propensity score matching and instrumental variables, are likely to be useful, although these methods cannot completely deal with unmeasured variables $^{32,33}$. However, most of the reviews included in the present study did not mention the implementation of adjustment in detail. 
Recently, several risk of bias appraisal tools for evaluating the quality of systematic reviews of observational studies in multiple domains have been developed, including ACROBAT-NRSI ${ }^{34-36}$. However, the present study showed that these tools are not yet widely implemented. Most of the studies reported the risk of bias using the NOS, although the NOS has been reported to show uncertain reliability and validity in previous studies 37,38 .

In the present study, we compared the effect estimates between observational studies and RCTs in metaanalyses combining both types of studies using two analytical methods: ROR and discrepancies in statistical significance between the study designs. The ROR with the $95 \% \mathrm{Cl}$ revealed that effect estimates were, on average, consistent between the two study designs. However, with regard to the interpretation of the findings, almost half of records showed discrepancies in statistical significance between the study designs. Further, $35.7 \%$ of records indicated disagreement in judgement between the two analytical methods. It might be reasonable to combine different types of designs in meta-analyses based on the ROR, because improvement of the statistical power leads to a more definite assessment if a sufficient number of RCTs cannot be obtained. However, interpreting the findings should be done with care, as inconsistent findings due to the modification of the analytical methods might reflect poor internal validity between the study designs. Additionally, the present study failed to identify systematic reviewlevel factors associated with the discrepancies in statistical significance, including difference in sample sizes between study designs and adjustment with confounding factors. In the future, we need to explore risk factors at the level of primary studies, such as detail of adjustment techniques for confounding factors and presence of biases in observational studies.

Several limitations of our study should be mentioned. First, it is possible that we failed to include several gray-area studies or smaller studies, although we performed a comprehensive search. Second, we included similar research questions that were published by different authors, which might have led to overestimation. Third, to compare effect estimates between study designs, we substituted original outcome measures, such as the RR and HR, instead of the OR, if the number of events could not be determined from primary articles to calculate the OR, similarly to previous studies ${ }^{21,39}$. However, results using the RR and HR are not necessarily consistent with those using the $\mathrm{OR}$, particularly when the number of events is large. Fourth, we were unable to estimate the ROR adjusted for the methodological quality of systematic reviews based on the AMSTAR tool, as almost all reviews were judged to be of low quality. Finally, because we sampled meta-analyses including both observational studies and RCTs, it is conceivable that extreme results, either from observational studies or from RCTs, could have been excluded when the original meta-analysis was being conducted, leading to spurious greater concordance between the two study designs. Without a pre-specified protocol, we cannot assess the extent of such practices.

\section{Conclusion}

This study indicates that evidence synthesis based on observational studies has been increasing in nephrology. When we examined ROR, we found no systematic differences in effect estimates between 
observational studies and RCTs when meta-analyses included both study designs. These findings might encourage researchers to justify the inclusion of observational studies in meta-analyses. This approach can increase statistical power and allow stronger causal inference. However, caution is needed when interpreting the findings from both observational studies and RCTs because the interpretations based on statistical significance were shown to be less concordant than those based on ROR. Further studies are necessary to explore the causes of these contradictions.

\section{Declarations}

\section{Data availability}

The datasets used and/or analysed during the current study are available from the corresponding author on reasonable request.

\section{Acknowledgements}

We sincerely appreciate the members of the Research Group on Meta-epidemiology at The Kyoto University School of Public Health (Tomoko Fujii, Yuki Kataoka, Yan Luo, Kenji Omae, Yasushi Tsujimoto, and Yusuke Tsutsumi) for their valuable advices.

\section{Author contributions}

M.K., A.O., A.T., K.K., and T.A.F. contributed to the research conception and design; M.K., A.O., A.T., and K.K. contributed to data extraction; M.K., A.O., A.T., K.K., and T.A.F. contributed to data analysis and interpretation: Each author contributed important intellectual content during manuscript drafting or revision and accepts personal accountability for the overall work, and agrees to ensure that questions pertaining to the accuracy or integrity of any portion of the work are appropriately investigated and resolved.

\section{Funding}

A.O. reports personal fees from Chugai, personal fees from Ono Pharmaceutical, personal fees from Eli Lilly, personal fees from Mitsubishi-Tanabe, personal fees from Asahi-Kasei, personal fees from Takeda, personal fees from Phyzer, grants from Advantest, outside the submitted work; A.T. reports personal fees from Mitsubishi-Tanabe, personal fees from Dainippon-Sumitomo, personal fees from Otsuka, outside the submitted work; T.A.F. reports grants and personal fees from Mitsubishi-Tanabe, personal fees from MSD, personal fees from Shionogi, outside the submitted work; M.K. and K.K. declare that they have no relevant financial interests.

\section{Competing interests}

The authors declare no competing interests. 


\section{References}

1. Nardini C. The ethics of clinical trials. 8, 387 (2014).

2. Black N. Why we need observational studies to evaluate the effectiveness of health care. 312,1215 1218 (1996).

3. Egger M, Schneider M, Davey Smith G. Spurious precision? Meta-analysis of observational studies. 316, 140-144 (1998).

4. Barton S. Which clinical studies provide the best evidence? The best RCT still trumps the best observational study. 321, 255-256 (2000).

5. Strippoli GF, Craig JC, Schena FP. The number, quality, and coverage of randomized controlled trials in nephrology. J Am Soc Nephrol. 15, 411-419 (2004).

6. Samuels JA, Molony DA. Randomized controlled trials in nephrology: state of the evidence and critiquing the evidence. Adv Chronic Kidney Dis. 19, 40-46 (2012).

7. Campbell MK, et al. Evidence-based medicine in nephrology: identifying and critically appraising the literature. Nephrol Dial Transplant. 15, 1950-1955 (2000).

8. Palmer SC, Sciancalepore M, Strippoli GF. Trial quality in nephrology: how are we measuring up? Am J Kidney Dis. 58, 335-337 (2011).

9. Charytan D, Kuntz RE. The exclusion of patients with chronic kidney disease from clinical trials in coronary artery disease. Kidney Int. 70, 2021-2030 (2006).

10. Deo A, Schmid CH, Earley A, Lau J, Uhlig K. Loss to analysis in randomized controlled trials in CKD. Am J Kidney Dis. 58, 349-355 (2011).

11. Garg AX, Hackam D, Tonelli M. Systematic review and meta-analysis: when one study is just not enough. Clin J Am Soc Nephrol. 3, 253-260 (2008).

12. Norris SL, et al. Observational studies in systematic [corrected] reviews of comparative effectiveness: AHRQ and the Effective Health Care Program. J Clin Epidemiol. 6, 1178-1186 (2011).

13. Guyatt GH, et al; The GRADE Working Group. GRADE guidelines: 9. Rating up the quality of evidence. J Clin Epidemiol. 64, 1311-1316 (2011).

14. Reeves BC, et al. An introduction to methodological issues when including non-randomised studies in systematic reviews on the effects of interventions. Res Synth Methods. 4, 1-11 (2013).

15. Greene T. Randomized and observational studies in nephrology: how strong is the evidence? Am J Kidney Dis. 53, 377-388 (2009).

16. Ray JG. Evidence in upheaval: incorporating observational data into clinical practice. Arch Intern Med. 162, 249-254 (2002).

17. Klein-Geltink JE, Rochon PA, Dyer S, Laxer M, Anderson GM. Readers should systematically assess methods used to identify, measure and analyze confounding in observational cohort studies. $J$ Clin Epidemiol. 60, 766-772 (2007). 
18. Kuss O, Legler T, Borgermann J. Treatments effects from randomized trials and propensity score analyses were similar in similar populations in an example from cardiac surgery. $\mathrm{J}$ Clin Epidemiol. 64, 1076-1084 (2011).

19. Lonjon $\mathrm{G}$, et al. Comparison of treatment effect estimates from prospective nonrandomized studies with propensity score analysis and randomized controlled trials of surgical procedures. Ann Surg. 259, 18-25 (2014).

20. Tzoulaki I, Siontis KC, loannidis JP. Prognostic effect size of cardiovascular biomarkers in datasets from observational studies versus randomised trials: meta-epidemiology study. 343, d6829 (2011).

21. Anglemyer A, Horvath HT, Bero L. Healthcare outcomes assessed with observational study designs compared with those assessed in randomized trials. Cochrane Database Syst Rev., MR000034 (2014).

22. Sterne JA, et al. Statistical methods for assessing the in uence of study characteristics on treatment e ects in 'meta-epidemiological' research. Med. 21, 1513-1524 (2002).

23. Higgins JPT, Green S. Cochrane Handbook for Systematic Reviews of Interventions Version 5.1.0 [updated March 2011]. The Cochrane Collaboration, cochrane-handbook.org (2011).

24. Golder S, Loke YK, Bland M. Meta-analyses of adverse effects data derived from randomised controlled trials as compared to observational studies: methodological overview. PLoS Med. 8 , e1001026 (2011).

25. Shea BJ, et al. AMSTAR 2: a critical appraisal tool for systematic reviews that include randomised or non-randomised studies of healthcare interventions, or both. 358, j4008 (2017).

26. Tajika A, Ogawa Y, Takeshima N, Hayasaka Y, Furukawa TA. Replication and contradiction of highly cited research papers in psychiatry: 10-year follow-up. Br J Psychiatry. 207, 357-362 (2015).

27. Sterne JA, et al. Statistical methods for assessing the influence of study characteristics on treatment effects in 'meta-epidemiological' research. Stat Med. 21, 1513-1524 (2002).

28. Deeks JJ, et al.; International Stroke Trial Collaborative Group; European Carotid Surgery Trial Collaborative Group. Evaluating non-randomised intervention studies. Health Technol Assess. 7, 囚-区, 1-173 (2003).

29. Kyriacou DN, Lewis RJ. Confounding by Indication in Clinical Research. 316, 1818-1819 (2016).

30. Rothman KJ, Greenland S, Lash TL. Modern Epidemiology $3^{\text {rd }}$ Wolters Kluwer, Lippincott Williams \& Wilkins, Philadelphia, 183-209 (2008).

31. Patel CJ, Burford B, loannidis JP. Assessment of vibration of effects due to model specification can demonstrate the instability of observational associations. J Clin Epidemiol. 68, 1046-1058 (2015).

32. Ripollone JE, Huybrechts KF, Rothman KJ, Ferguson RE, Franklin JM. Implications of the propensity score matching paradox in Am J Epidemiol. 187, 1951-1961 (2018).

33. Staffa SJ, Zurakowski D. Five steps to successfully implement and evaluate propensity score matching in clinical research studies. Anesth Analg. 127, 1066-1073 (2018). 
34. Sterne JA, et al. ROBINS-I: a tool for assessing risk of bias in non-randomised studies of interventions. 355, i4919 (2016).

35. Viswanathan M, Berkman ND. Development of the RTI item bank on risk of bias and precision of observational studies. J Clin Epidemiol. 65, 163-178 (2012).

36. Sterne JAC, Higgins JPT, Reeves BC on behalf of the development group for ACROBAT- NRSI. A Cochrane Risk Of Bias Assessment Tool: for Non-Randomized Studies of Interventions (ACROBATNRSI), Version 1.0.0, http://www.riskofbias.info. (2014).

37. Wells GA, et al. The Newcastle-Ottawa Scale (NOS) for assessing the quality of nonrandomised studies in meta-analysis. Ottawa, Canada: University of

Ottawa. http://www.ohri.ca/programs/clinical_epidemiology/oxford.asp.

38. Lo CK, Mertz D, Loeb M. Newcastle-Ottawa Scale: comparing reviewers' to authors' assessments. BMC Med Res Methodol. 14, 45 (2014).

39. Hayden JA, Co^te'P, Bombardier C. Evaluation of the quality of prognosis studies in systematic reviews. Ann Intern Med. 144, 427-437 (2006).

40. Downs SH, Black N. The feasibility of creating a checklist for the assessment of the methodological quality both of randomised and non-randomised studies of health care interventions. J Epidemiol Commnity Health. 52, 377-384 (1998).

41. Harris RP, et al.; Methods Work Group, Third US Preventive Services Task Force. Methods Work Group, Third US Preventive Services Task Force. Current Methods of the U.S. Preventive Services Task Force A Review of the Process. Am J Prev Med. 20, 21-35 (2001).

\section{Tables}

Table 1. Baseline characteristics of the 477 included systematic reviews of observational studies 


\begin{tabular}{|c|c|}
\hline \multicolumn{2}{|l|}{ Characteristics } \\
\hline Number of primary studies & $13(9$ to 21$)$ \\
\hline \multicolumn{2}{|l|}{ Cause of kidney injury } \\
\hline Renal tumor & $75(15.7 \%)$ \\
\hline End stage renal disease & $68(14.3 \%)$ \\
\hline Renal transplant & $39(8.2 \%)$ \\
\hline AKILCIN & $50(10.5 \%)$ \\
\hline Nephrotoxin & $9(1.9 \%)$ \\
\hline Chronic glomerulonephritis & $11(2.3 \%)$ \\
\hline Diabetic nephropathy & $14(2.9 \%)$ \\
\hline Kidney stone & $14(2.9 \%)$ \\
\hline Basic Research (genetic, molecular, others) & $82(17.2 \%)$ \\
\hline Children or pregnant women with renal diseases & $9(1.9 \%)$ \\
\hline CKD (complication, risk factor, therapy, outcome research, others) & $99(20.8 \%)$ \\
\hline Others (RDN, renal artery, others) & $7(1.5 \%)$ \\
\hline \multicolumn{2}{|l|}{ Included studies } \\
\hline RCT & $114(23.9 \%)$ \\
\hline Clinical trials (nonrandomized studies) & $18(3.8 \%)$ \\
\hline Cohort studies & $320(67.1 \%)$ \\
\hline Case-control studies & $161(33.8 \%)$ \\
\hline Cross sectional studies & $52(10.9 \%)$ \\
\hline Case series & $5(1.1 \%)$ \\
\hline Before-after studies & $7(1.5 \%)$ \\
\hline Unclear observational studies & $54(11.3 \%)$ \\
\hline \multicolumn{2}{|l|}{ Funding support } \\
\hline Yes & $210(44.0 \%)$ \\
\hline No & $102(21.4 \%)$ \\
\hline Unclear & $165(34.6 \%)$ \\
\hline Risk of bias in observational studies & \\
\hline
\end{tabular}




\begin{tabular}{|l|c|}
\hline NOS & $183(38.4 \%)$ \\
\hline Tool by Hayden JA.et al [39] & $14(2.9 \%)$ \\
\hline Tool by Downs and Black [40] & $13(2.7 \%)$ \\
\hline Tool by USPSTF/Task Force [41] & $11(2.3 \%)$ \\
\hline ACROBAT NRSI ${ }^{1}$ & $4(0.8 \%)$ \\
\hline Others & $38(8.0 \%)$ \\
\hline Unclear & $26(5.5 \%)$ \\
\hline None & $188(39.4 \%)$ \\
\hline Reporting assessment & \\
\hline Yes & $227(47.6 \%)$ \\
\hline No & $250(52.4 \%)$ \\
\hline
\end{tabular}

Results of continuous variables are shown as the mean (standard deviation) or median (interquartile range).

AKI, acute kidney injury; CIN, contrast induced nephropathy; CKD, chronic kidney disease; RCT, randomized controlled trial; NOS, Newcastle-Ottawa Scale; USPSTF/Task Force, The U.S. Preventive Services Task Force; ACROBAT-NRSI, A Cochrane Risk of Bias Assessment Tool for Non-Randomized Studies of Interventions

Table 2. Baseline characteristics of the 56 included meta-analyses combining both observational studies and randomized control trials 


\begin{tabular}{|c|c|c|}
\hline total & $\begin{array}{l}\text { No discrepancy } \\
\text { (significant in } \\
\text { the same } \\
\text { direction) } \\
(n=14)\end{array}$ & $\begin{array}{l}\text { No } \\
\text { discrepancy } \\
\text { (both non- } \\
\text { significant) } \\
(n=15)\end{array}$ \\
\hline
\end{tabular}

Discrepancy

(one significant and the other nonsignificant) $(n=27)$

Number of primary studies

Overall studies

11

(6.5 to

12 (5 to 15$)$

7 (4 to 11$)$

12 (9 to 16$)$

14.5)

Observational studies

$7(2.5$

4.5 (1 to 11$)$

4 (2 to 8$)$

9 (5 to 13)

RCTs

3 (2 to 2.5 (1 to 9$)$

2 (2 to 4$)$

3 (2 to 5)

5)

Countries, \%

China/Taiwan

$21 \quad 4(28.6)$

(37.5)

USA

17

6 (42.9)

$3(20.0)$

8 (29.6)

European countries

10

$1(7.1)$

5 (33.3)

4 (14.8)

Others

8

(14.3)

3 (21.4)

$1(6.7)$

4 (14.8)

Publication years, n (\%)

2012 or before

10
$(17.9)$

(17.9)

2014-2013

15

(26.8)

$1(7.1)$

2 (13.3)

7 (25.9)

2015-2016

31

(55.4)

$3(21.4)$

$4(26.7)$

8 (29.6)

Cause of kidney injury, n (\%)

Renal tumor

4

(7.1)

10 (71.4)

$9(60.0)$

$12(44.4)$

End stage renal disease

\section{1}

(19.6)

Renal transplant

AKI $\mathrm{CIN}$
7

(12.5)

17

(30.4)
$1(7.1)$

2 (13.3)

2 (14.3)

4 (28.6)

2 (14.3)

$4(26.7)$

$11(40.7)$ 


\begin{tabular}{|c|c|c|c|c|}
\hline Nephrotoxin & $\begin{array}{l}2 \\
\text { (3.6) }\end{array}$ & $0(0)$ & $2(13.3)$ & $0(0)$ \\
\hline Chronic glomerulonephritis & $\begin{array}{l}1 \\
(1.8)\end{array}$ & $0(0)$ & $0(0)$ & $1(3.7)$ \\
\hline Diabetic nephropathy & $\begin{array}{l}1 \\
(1.8)\end{array}$ & $1(7.1)$ & $0(0)$ & $0(0)$ \\
\hline Kidney stone & $\begin{array}{l}6 \\
(10.7)\end{array}$ & $2(14.3)$ & $4(26.7)$ & $0(0)$ \\
\hline $\begin{array}{l}\text { Children or pregnant women } \\
\text { with renal diseases }\end{array}$ & $\begin{array}{l}1 \\
(1.8)\end{array}$ & $1(7.1)$ & $0(0)$ & $0(0)$ \\
\hline $\begin{array}{l}\text { CKD (complication, risk factor, } \\
\text { therapy, outcome research, } \\
\text { others) }\end{array}$ & $\begin{array}{l}6 \\
(10.7)\end{array}$ & $1(7.1)$ & $1(6.7)$ & $4(14.8)$ \\
\hline \multicolumn{5}{|l|}{ Included studies, n (\%) } \\
\hline Cohort studies & $\begin{array}{l}45 \\
(80.4)\end{array}$ & $11(78.6)$ & $13(86.7)$ & $21(77.8)$ \\
\hline Case-control studies & $\begin{array}{l}7 \\
(12.5)\end{array}$ & $1(7.1)$ & $3(20.0)$ & $3(11.1)$ \\
\hline Cross-sectional studies & $\begin{array}{l}3 \\
(5.4)\end{array}$ & $0(0)$ & $1(6.7)$ & $2(7.4)$ \\
\hline Unclear observational studies & $\begin{array}{l}11 \\
(19.6)\end{array}$ & $4(28.6)$ & $0(0)$ & $7(25.9)$ \\
\hline Other non randomized studies & $\begin{array}{l}5 \\
(8.9)\end{array}$ & $0(0)$ & $2(13.3)$ & $3(11.1)$ \\
\hline Subjective outcomes, n (\%) & $\begin{array}{l}56 \\
(100)\end{array}$ & $14(100)$ & $15(100)$ & $27(100)$ \\
\hline $\begin{array}{l}\text { Pharmacological interventions, } \\
\text { n (\%) }\end{array}$ & $\begin{array}{l}18 \\
(32.1)\end{array}$ & $5(35.7)$ & $6(40.0)$ & $7(25.9)$ \\
\hline \multicolumn{5}{|l|}{ Funding support for SR, n (\%) } \\
\hline Yes & $\begin{array}{l}22 \\
(39.3)\end{array}$ & $10(71.4)$ & $3(20.0)$ & $9(33.3)$ \\
\hline No & $\begin{array}{l}16 \\
(28.6)\end{array}$ & $2(14.3)$ & $7(46.7)$ & $7(25.9)$ \\
\hline unclear & $\begin{array}{l}18 \\
(32.1)\end{array}$ & $2(14.3)$ & $5(33.3)$ & $11(40.7)$ \\
\hline \multicolumn{5}{|l|}{$\begin{array}{l}\text { Risk of bias in observational } \\
\text { studies, } \mathrm{n}(\%)\end{array}$} \\
\hline NOS & $\begin{array}{l}25 \\
(44.6)\end{array}$ & $9(64.3)$ & $7(46.7)$ & $9(33.3)$ \\
\hline
\end{tabular}




\begin{tabular}{|lllll|}
\hline Downs and Black & $\begin{array}{l}4 \\
(7.1)\end{array}$ & $0(0)$ & $1(6.7)$ & $3(11.1)$ \\
\hline ACROBAT NRSI & $\begin{array}{l}1 \\
(1.8)\end{array}$ & $0(0)$ & $0(0)$ & $1(3.7)$ \\
\hline Others & $\begin{array}{l}10 \\
(17.9)\end{array}$ & $1(7.1)$ & $2(13.3)$ & $7(25.9)$ \\
\hline Unclear & $\begin{array}{l}2 \\
(3.6)\end{array}$ & $0(0)$ & $0(0)$ & $2(7.4)$ \\
\hline None & $\begin{array}{l}14 \\
(25.0)\end{array}$ & $4(28.6)$ & $5(33.3)$ & $5(18.5)$ \\
\hline $\begin{array}{l}\text { Existence of reporting } \\
\text { assessment, } \mathrm{n}(\%)\end{array}$ & $\begin{array}{l}32 \\
(57.1)\end{array}$ & $7(50.0)$ & $6(40.0)$ & $19(70.4)$ \\
\hline
\end{tabular}

Results of continuous variables are shown as the mean (standard deviation) or median (interquartile range).

RCT, randomized controlled trial; USA, United States of America; AKI, acute kidney injury; CIN, contrast induced nephropathy; CKD, chronic kidney disease; NOS, Newcastle-Ottawa Scale; USPSTF/Task Force, The U.S. Preventive Services Task Force; ACROBAT-NRSI, A Cochrane Risk of Bias Assessment Tool for Non-Rando

Table 3. Predictors of discrepancies in results between observational studies and randomized control trials 


\begin{tabular}{|c|c|c|c|}
\hline \multirow[b]{2}{*}{$\begin{array}{l}\text { Difference in number of papers between observational studies and } \\
\text { RCTs }\end{array}$} & \multicolumn{2}{|c|}{ OR $(95 \% \mathrm{Cl})$} & \multirow{2}{*}{$\begin{array}{l}p- \\
\text { value } \\
0.085\end{array}$} \\
\hline & 1.09 & $\begin{array}{l}(0.99 \text { to } \\
1.21)\end{array}$ & \\
\hline \multicolumn{4}{|l|}{ Countries } \\
\hline China/Taiwan & 1.04 & $\begin{array}{l}(0.25 \text { to } \\
4.28)\end{array}$ & 0.96 \\
\hline USA & \multicolumn{3}{|c|}{ Reference } \\
\hline European countries & 0.63 & $\begin{array}{l}(0.10 \text { to } \\
3.89)\end{array}$ & 0.62 \\
\hline Other countries & 1.11 & $\begin{array}{l}(0.17 \text { to } \\
7.33)\end{array}$ & 0.91 \\
\hline \multicolumn{4}{|l|}{ Publication year } \\
\hline 2012 or before & \multicolumn{3}{|c|}{ Reference } \\
\hline 2013-2014 & 0.59 & $\begin{array}{l}(0.095 \text { to } \\
3.64)\end{array}$ & 0.57 \\
\hline 2015-2016 & 0.45 & $\begin{array}{l}(0.087 \text { to } \\
2.34)\end{array}$ & 0.34 \\
\hline Pharmacological interventions (vs. none) & 1.12 & $\begin{array}{l}(0.27 \text { to } \\
4.64)\end{array}$ & 0.87 \\
\hline Adjustment for confounding factors & 0.59 & $\begin{array}{l}(0.14 \text { to } \\
2.49)\end{array}$ & 0.48 \\
\hline
\end{tabular}

OR, odds ratio; $\mathrm{Cl}$, confidence interval; USA, United States of America

Adjusted for differences in the number of primary articles between observational studies and RCTs, publication year, country of first author, and pharmacological intervention.

Table 4. Comparison of ROR and discrepancies defined by statistical significance 


\begin{tabular}{|l|lll|}
\hline \multicolumn{5}{|c|}{ Discrepancy in the interpretations based on statistical significance } \\
\hline ROR & $\begin{array}{l}\text { No discrepancy (significant } \\
\text { in the same direction) } \\
(n=14)\end{array}$ & $\begin{array}{l}\text { No } \\
\text { discrepancy } \\
\text { (both non- } \\
\text { significant) } \\
(n=15)\end{array}$ & $\begin{array}{l}\text { Discrepancy (one significant and } \\
\text { the other non-significant) } \\
(n=27)\end{array}$ \\
$\begin{array}{l}p<0.05 \\
(n=8)\end{array}$ & $1(1.8)$ & $0(0)$ & $7(12.5)$ \\
$\begin{array}{c}p \\
(n=05\end{array}$ & $13(23.2)$ & $15(26.8)$ & $20(35.7)$ \\
\hline
\end{tabular}

ROR, ratio of odds ratio $₫$ Number (\%)

\section{Figures}

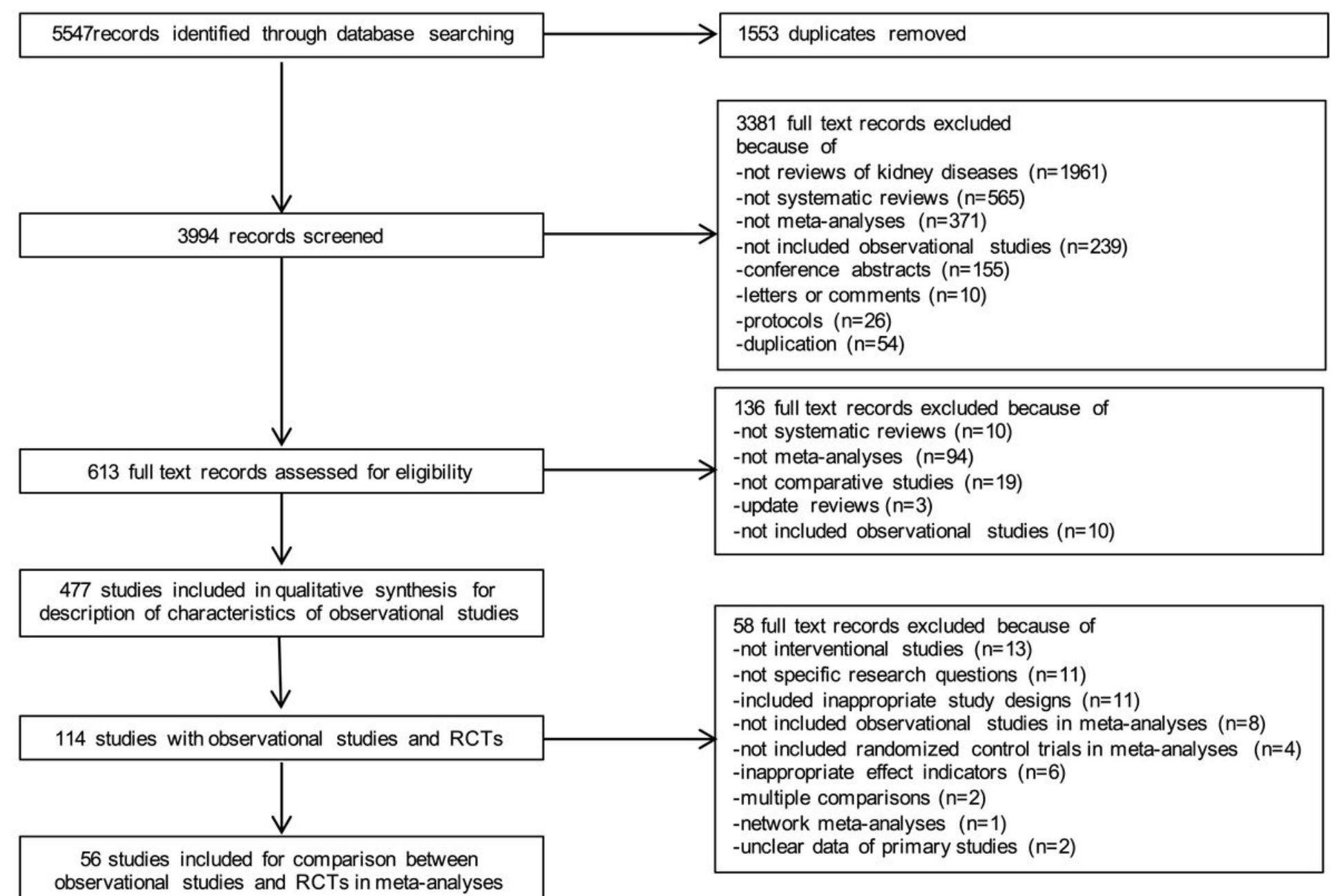

\section{Figure 1.}




\begin{tabular}{|c|c|}
\hline 5547records identified through database searching & 1553 duplicates removed \\
\hline$\downarrow$ & \multirow{3}{*}{$\begin{array}{l}3381 \text { full text records excluded } \\
\text { because of } \\
\text {-not reviews of kidney diseases }(n=1961) \\
\text {-not systematic reviews }(n=565) \\
\text {-not meta-analyses ( } n=371) \\
\text {-not included observational studies }(n=239) \\
\text {-conference abstracts }(n=155) \\
\text {-letters or comments }(n=10) \\
\text {-protocols }(n=26) \\
\text {-duplication }(n=54)\end{array}$} \\
\hline 3994 records screened & \\
\hline & \\
\hline$\downarrow$ & $\begin{array}{l}136 \text { full text records excluded because of } \\
\text {-not systematic reviews }(n=10)\end{array}$ \\
\hline 613 full text records assessed for eligibility & -not comnarative studies $(n=19)$ \\
\hline & $\begin{array}{l}\text {-update reviews }(n=3) \\
\text {-not included observational studies }(n=10)\end{array}$ \\
\hline $\begin{array}{l}477 \text { studies included in qualitative synthesis for } \\
\text { description of characteristics of observational studies }\end{array}$ & \\
\hline$\downarrow$ & $\begin{array}{l}\text {-not interventional studies }(n=13) \\
\text {-included inappropriate study designs }(n=11)\end{array}$ \\
\hline 114 studies with observational studies and RCTs & $\begin{array}{l}\text {-not included observational studies in meta-analyses }(n=8) \\
\text {-not included randomized control trials in meta-analyses }(n=4)\end{array}$ \\
\hline$\Downarrow$ & $\begin{array}{l}\text {-inappropriate effect indicators }(n=6) \\
\text {-multiple comparisons }(n=2) \\
\text {-network meta-analyses }(n=1)\end{array}$ \\
\hline 56 studies included for comparison between & -unclear data of primary studies $(n=2)$ \\
\hline
\end{tabular}

\section{Figure 1.}

\section{Figure 1}

Study flow diagram and study selection process 


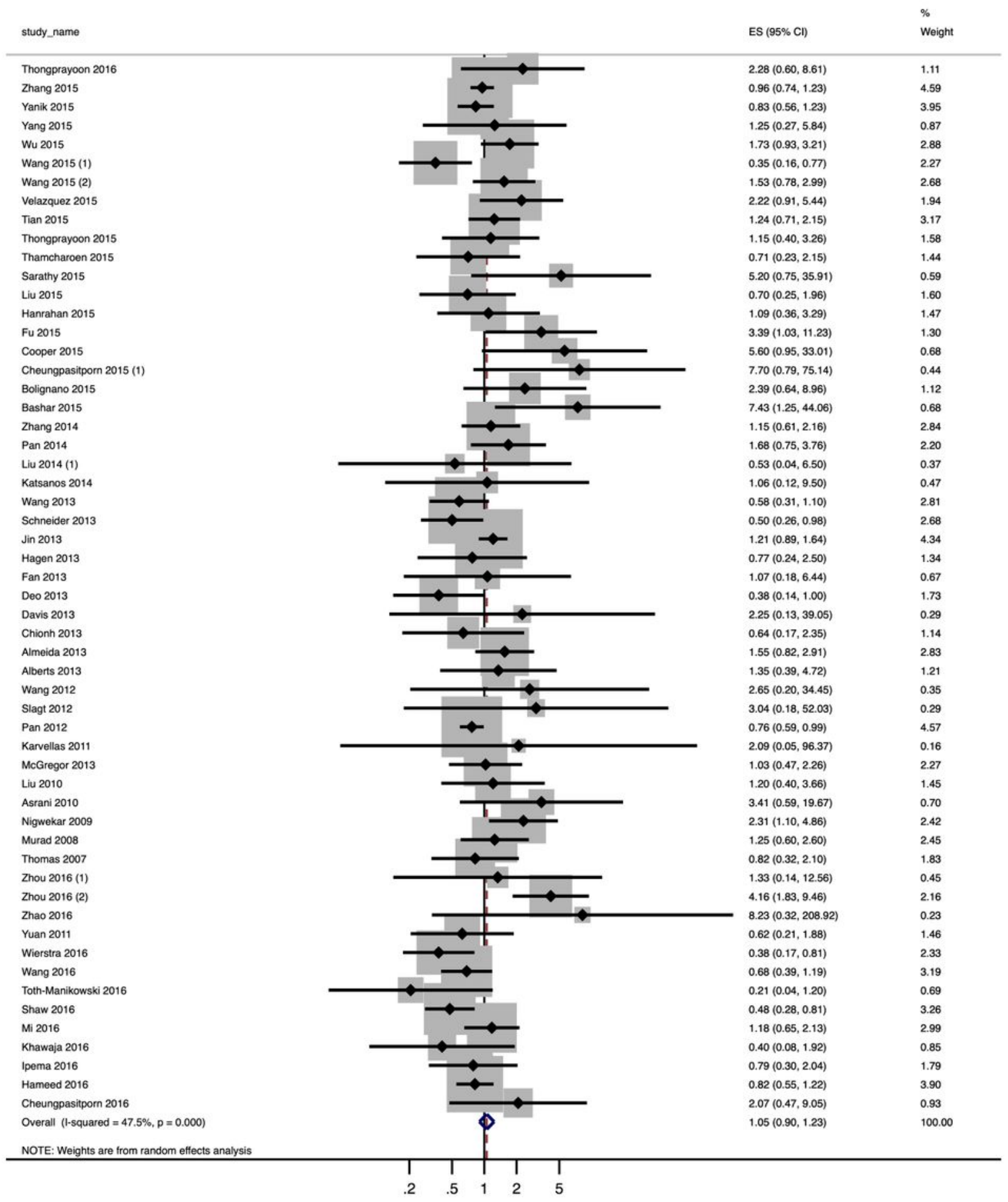

Figure 2.

Figure 2

Forest plot of pooled ROR with $95 \% \mathrm{Cl}$ that assessed the systematic differences in the effect estimates between observational studies and RCTs in meta-analyses combining both types of studies in nephrology. ROR, ratio of odds ratio; $95 \% \mathrm{Cl}, 95 \%$ confidence interval; RCT, randomized controlled trial 


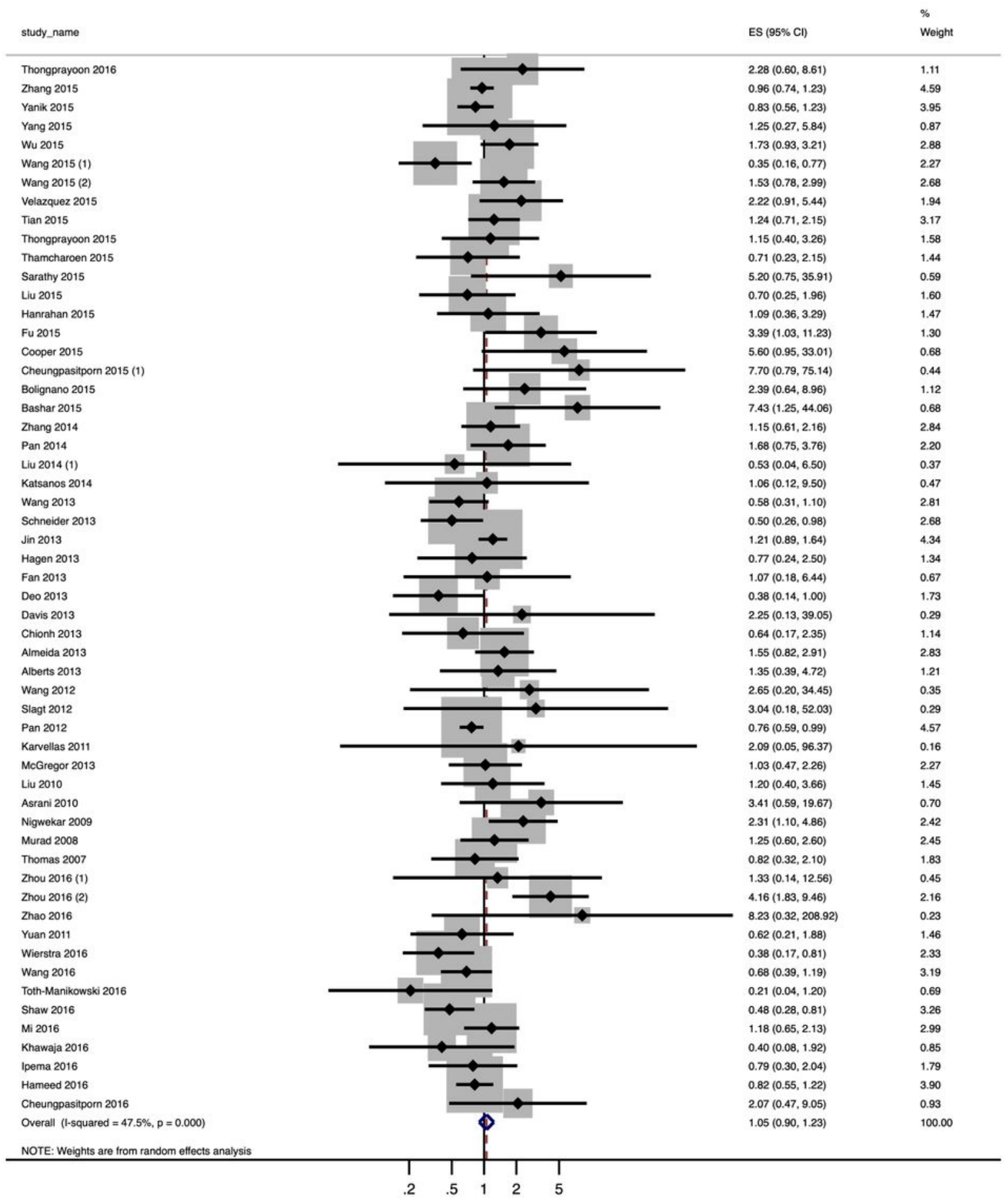

Figure 2.

Figure 2

Forest plot of pooled ROR with $95 \% \mathrm{Cl}$ that assessed the systematic differences in the effect estimates between observational studies and RCTs in meta-analyses combining both types of studies in nephrology. ROR, ratio of odds ratio; $95 \% \mathrm{Cl}, 95 \%$ confidence interval; RCT, randomized controlled trial 
This is a list of supplementary files associated with this preprint. Click to download.

- SupplementTable14.doc

- SupplementTable14.doc 